\title{
Proceedings of the 32nd Symposium on Implementation and Application of Functional Languages IFL 2020
}

Canterbury, United Kingdom online

2nd - 4th September 2020

Edited by

Olaf Chitil

ACM ISBN 978-1-45-3-8963-1

\section{ICPS}

Published by $A C M$

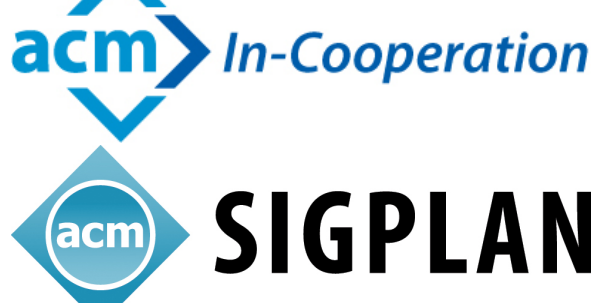

University of

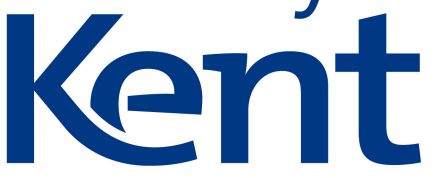


The Association for Computing Machinery

1601 Broadway, 10th Floor New York New York 10019-7434

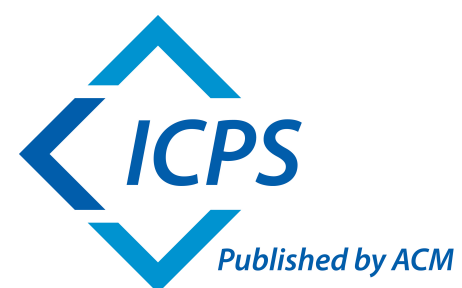

ACM ISBN 978-1-45-3-8963-1

ACM COPYRIGHT NOTICE. Copyright 2021 by the Association for Computing Machinery, Inc. Permission to make digital or hard copies of part or all of this work for personal or classroom use is granted without fee provided that copies are not made or distributed for profit or commercial advantage and that copies bear this notice and the full citation on the first page. Copyrights for components of this work owned by others than ACM must be honored. Abstracting with credit is permitted. To copy otherwise, to republish, to post on servers, or to redistribute to lists, requires prior specific permission and/or a fee. Request permissions from Publications Dept., ACM, Inc., fax +1 (212) 869-0481, or permissions@acm.org.

For other copying of articles that carry a code at the bottom of the first or last page, copying is permitted provided that the per-copy fee indicated in the code is paid through the Copyright Clearance Center, 222 Rosewood Drive, Danvers, MA 01923, +1-978-750-8400, +1-978-750-4470 (fax). 


\section{Preface}

The goal of the IFL symposia is to bring together researchers actively engaged in the implementation and application of functional and function-based programming languages.

This volume contains the proceedings of IFL 2020, which was held in the period 2nd - 4th September 2020. Originally the symposium was planned to take place on the Canterbury campus of the University of Kent in Canterbury, UK. However, because of the Covid-19 pandemic, it was changed to a virtual online event with presentations, discussions and social gatherings all done via the online video meeting system Zoom.

The chairs of IFL 2019, Jurrien Stutterheim (Standard Chartered Bank Singapore) and Wei Ngan Chin (National University of Singapore), gave their financial surplus to IFL 2020. Together with free administrative and technical support from the University of Kent, especially by technician Daniel Knox, this enabled free participation at IFL 2020. Thus a record number of 156 people registered and up to 80 people at a time participated on Zoom.

Following IFL tradition, IFL 2020 used a post-symposium review process to produce the formal proceedings. Before the symposium authors submitted draft papers via EasyChair. These draft papers were only screened by the program chair to make sure that they are within the scope of IFL. The draft papers were made available to all participants at the symposium. 28 papers were presented at the symposium. After the symposium every presenter was invited to submit a full paper, incorporating feedback from discussions at the symposium. Again via EasyChair 20 papers were submitted of which 14 were accepted by the program committee for these final proceedings. Reviewing was single blind with at least 3 reviewers per paper.

The programme committee also selected the best paper presented at IFL 2020 and awarded it the Peter Landin Prize. The winners are

Kavon Farvardin and John Reppy

with their paper

A New Backend for Standard ML of New Jersey

The prize, which carries a cash award equivalent to $€ 150$, is provided by Greg Michaelson and Kevin Hammond.

Finally, a (virtual) meeting of the steering committee took place at the symposium. After more than 30 years the founder of IFL, Rinus Plasmeijer, stepped down as steering committee chair. The steering committee elected Jurriaan Hage as new chair and Pieter Koopman as new publicity chair. IFL 2021 will be organised by Pieter Koopman and Peter Achten, Radboud University, The Netherlands.

Olaf Chitil, Chair

Canterbury, June 2021 


\section{Program Committee}

Kenichi Asai

Olaf Chitil

Martin Erwig

Dániel Horpácsi

Zhenjiang $\mathrm{Hu}$

Hans-Wolfgang Loidl

Neil Mitchell

Marco T. Morazán

Rinus Plasmeijer

Colin Runciman

Mary Sheeran

Josep Silva

Jurrien Stutterheim

Josef Svenningsson

Peter Thiemann

Kanae Tsushima

Marcos Viera

Janis Voigtländer
Ochanomizu University, Japan

University of Kent, United Kingdom

Oregon State University, United States

Eötvös Loránd University, Hungary

Peking University, China

Heriot-Watt University, United Kingdom

Facebook, United Kingdom

Seton Hall University, United States

Radboud University, Netherlands

University of York, United Kingdom

Chalmers University of Technology, Sweden

Universitat Politècnica de València, Spain

Standard Chartered, Singapore

Facebook, United Kingdom

University of Freiburg, Germany

National Institute of Informatics, Japan.

Universidad de la República, Montevideo, Uruguay

University of Duisburg-Essen, Germany

\section{Additional Reviewers}

Divya Bajaj

Péter Bereczky

Danila Fedorin

Jurriaan Hage

Pieter Koopman

Prashant Kumar

Dániel Lukács

Fernando Rubio

Bo Joel Svensson

Oliver Westphal

Ivo Gabe de Wolff 


\section{Table of Contents}

Building an Integrated Development Environment (IDE) on top of a Build System Neil Mitchell, Moritz Kiefer, Pepe Iborra, Luke Lau, Zubin Duggal, Hannes Siebenhandl, Javier Neira Sanchez, Matthew Pickering and Alan Zimmerman

HoCL: High level specification of dataflow graphs Jocelyn Serot

End-user feedback in multi-user workflow systems Nico Naus and Johan Jeuring

Heuristics-based Type Error Diagnosis for Haskell — The case of GADTs and local reasoning

Joris Burgers, Jurriaan Hage and Alejandro Serrano

A Declarative Gradualizer with Language Transformations

Benjamin Mourad and Matteo Cimini.

A New Backend for Standard ML of New Jersey

Kavon Farvardin and John Reppy

Template-based Theory Exploration: Discovering Properties of Functional Programs by Testing

Sólrún Halla Einarsdóttir, Nicholas Smallbone and Moa Johansson

Type- and Control-Flow Directed Defunctionalization

Maheen Riaz Contractor and Matthew Fluet

Zero-Cost Constructor Subtyping

Andrew Marmaduke, Christopher Jenkins and Aaron Stump

Resource Analysis for Lazy Evaluation with Polynomial Potential

Sara Moreira, Pedro Vasconcelos and Mário Florido

Typed Directional Composable Editors in iTasks

Bas Lijnse and Rinus Plasmeijer

Polymorphic System I

Cristian F. Sottile, Alejandro Daz-Caro and Pablo E. Martnez López

Effective Host-GPU Memory Management Through Code Generation

Hans-Nikolai Vießmann and Sven-Bodo Scholz

Validating Formal Semantics by Property-Based Cross-Testing

Péter Bereczky, Dániel Horpácsi, Judit Köszegi, Soma Szeier and Simon Thompson 